\title{
ESSENTIAL SPECTRA OF OPERATORS IN THE CLASS $\mathscr{B}_{n}(\Omega)^{1}$
}

\author{
KARIM SEDDIGHI
}

\begin{abstract}
For a connected open subset $\Omega$ of the plane and $n$ a positive integer, let $\mathscr{B}_{n}(\Omega)$ be the space introduced by Cowen and Douglas in their paper Complex Geometry and Operator Theory. Our paper deals with characterizing the essential spectrum of an operator $T$ in $\mathscr{G}_{n}(\Omega)$ for which $\sigma(T)=\bar{\Omega}$ and the point spectrum of $T^{*}$ is empty. This class of operators forms ar important part of $\mathscr{B}_{n}(\Omega)$ denoted by $\mathscr{B}_{n}^{\prime}(\Omega)$. We use this characterization to give another proof of the result of Axler, Conway and McDonald on determining the essential spectrum of the Bergman operator.

Let $A_{n}(G)=\left\{S: T=S^{*}\right.$ is in $\left.\mathscr{B}_{n}^{\prime}\left(G^{*}\right)\right\}$. We also characterize the weighted shifts in $A_{1}(G)$.
\end{abstract}

This paper deals with characterizing the essential spectrum of an operator $T$ in $\mathscr{B}_{n}(\Omega)$ for which $\sigma(T)=\bar{\Omega}$ and the point spectrum of $T^{*}$ is empty. This class of operators is denoted by $\mathscr{B}_{n}^{\prime}(\Omega)$. We use this characterization to give another proof of the result of Axler, Conway and McDonald on determining the essential spectrum of the Bergman operator.

Let $A_{n}(G)=\left\{S: T=S^{*}\right.$ is in $\left.\mathscr{B}_{n}^{\prime}\left(G^{*}\right)\right\}$. We also characterize the weighted shifts in $A_{1}(G)$.

NOTATION AND TERMINOLOGY. The following notation will be retained throughout the rest of this paper:

$$
\square=\text { empty set }, \quad \mathbf{C}=\text { complex plane. }
$$

For a subset $E$ of the plane, $\partial E$ is the boundary of $E$, int $E$ (or $E^{\circ}$ ) is the interior of $E$, and $\bar{E}$ is the closure of $E$.

$\mathcal{E}(\mathcal{H})$ will denote the collection of bounded linear operators on the complex Hilbert space $\mathcal{H}$.

For $T$ in $E(G)$, the spectrum of $T$, point spectrum of $T$, and approximate point spectrum of $T$ will be denoted by $\sigma(T), \sigma_{p}(T)$, and $\sigma_{a p}(T)$ respectively. See [4] for the appropriate definitions.

An operator $T$ in $\mathscr{L}(\mathcal{H})$ is called a Fredholm operator if $\operatorname{ran} T$ is closed and both $\operatorname{ker} T$ and $\operatorname{ker} T^{*}$ are finite dimensional. The essential spectrum of $T$, denoted by $\sigma_{e}(T)$, is the set of all $\lambda$ in $\mathbf{C}$ such that $T-\lambda$ is not Fredholm.

The closed linear span of a family of subspaces $\left\{\mathscr{H}_{\alpha}\right\}$ is $V_{\alpha} \mathcal{H}_{\alpha}$.

Received by the editors November 25, 1981, and, in revised form, June 1, 1982.

1980 Mathematics Subject Classification. Primary 47A53, 47B30: Secondary 47B37.

Key words and phrases. Essential spectrum, Bergman operator, weighted shift.

'The results in this paper are part of the author's $\mathrm{Ph} . \mathrm{D}$. thesis written at Indiana University under the direction of Professor John B. Conway. 
If $B$ is a subset of the complex plane, then $B^{*}=\{z: \bar{z} \in B\}$. Finally $B(z, \delta)=\{\lambda$ $\in \mathbf{C}:|z-\lambda|<\delta\}$.

1. Essential spectrum. For a connected open subset $\Omega$ of the plane and $n$ a positive integer, let $\mathscr{B}_{n}(\Omega)$ denote the operators $T$ defined on the Hilbert space $\mathcal{H}$ which satisfy

(a) $\Omega \subseteq \sigma(T)=\{\omega \in \mathrm{C}: T-\omega$ is not invertible $\}$,

(b) $\operatorname{ran}(T-\omega)=\mathcal{H}$ for $\omega$ in $\Omega$,

(c) $V_{\omega \in \Omega} \operatorname{ker}(T-\omega)=\mathcal{H}$, and

(d) $\operatorname{dim} \operatorname{ker}(T-\omega)=n$ for $\omega$ in $\Omega$.

The space $\mathscr{B}_{n}(\Omega)$ has been introduced and investigated by Cowen and Douglas [3].

In this section we want to determine the essential spectrum of operators in $\mathscr{B}_{n}^{\prime}(\Omega)$, where $\mathscr{B}_{n}^{\prime}(\Omega)=\left\{T \in \mathscr{B}_{n}(\Omega): \sigma(T)=\bar{\Omega}\right.$ and $\left.\sigma_{p}\left(T^{*}\right)=\square\right\}$, and, as a consequence, we deduce the result of Axler, Conway and McDonald [1] on characterizing the essential spectrum of the Bergman operator.

(1.1) ThEOREM. Let $\Omega$ be an open connected subset of the plane, let $n$ be a positive integer, and let $T \in \mathcal{L}(\mathcal{H})$ such that

(i) $\sigma(T)=\bar{\Omega}$,

(ii) $\operatorname{ran}(T-\omega)$ is closed, $\omega \in \Omega$,

(iii) $\operatorname{dim} \operatorname{ker}(T-\omega)=n, \omega \in \Omega$,

(iv) $\sigma_{p}\left(T^{*}\right)=\square$.

Let $\Omega_{0}=$ all points $\omega_{0} \in \bar{\Omega}$ such that $\operatorname{ran}\left(T-\omega_{0}\right)$ is closed and there exist holomorphic F-valued functions $\left\{e_{i}(\omega)\right\}_{i=1}^{n}$ defined on some neighborhood $B$ of $\omega_{0}$ such that for each $\omega$ in $B\left\{e_{i}(\omega)\right\}_{i=1}^{n}$ forms a basis for $\operatorname{ker}(T-\omega)$. Then $\sigma_{e}(T)=\partial \Omega_{0}$.

Proof. Let $\Omega^{\prime}=\left\{\omega_{0} \in \bar{\Omega}: \operatorname{ran}\left(T-\omega_{0}\right)\right.$ is closed and $\operatorname{dim} \operatorname{ker}(T-\omega)=n$ for $\omega$ in some neighborhood $B$ of $\left.\omega_{0}\right\}$. Note that $\Omega^{\prime}$ is open and $\Omega \subseteq \Omega^{\prime} \subseteq \bar{\Omega}$. Hence the closure of $\Omega^{\prime}$ is $\bar{\Omega}$. Also for $\omega \in \Omega^{\prime}, T-\omega$ is Fredholm and $\operatorname{ind}(T-\omega)=n$. We show that $\sigma_{e}(T)=\partial \Omega^{\prime}$ and $\Omega^{\prime}=\Omega_{0}$.

If $\omega \notin \partial \Omega^{\prime}$, either $\omega$ is not in the closure of $\Omega^{\prime}$ (hence $\omega \notin \bar{\Omega}$ ), in which case $T-\omega$ is invertible and hence Fredholm, or $\omega$ is in the closure of $\Omega^{\prime}$, in which case $\omega \in \Omega^{\prime}$ and $T-\omega$ is Fredholm. So $\sigma_{e}(T) \subseteq \partial \Omega^{\prime}$. If $\omega_{0} \notin \sigma_{e}(T)$, then $T-\omega_{0}$ is Fredholm; thus $T-\omega$ is Fredholm for $\omega$ in some neighborhood $B$ of $\omega_{0}$ and $\operatorname{ind}(T-\omega)$ is constant on this neighborhood. If $B \cap \Omega^{\prime}=\square$, then $\omega_{0} \notin \partial \Omega^{\prime}$. If $B \cap \Omega^{\prime} \neq \square$, then $\operatorname{ind}(T-\omega)=n, \omega \in B$. Because $\sigma_{p}\left(T^{*}\right)=\square, \operatorname{dim} \operatorname{ker}(T-\omega)=n, \omega \in B$. Thus $B \subseteq \Omega^{\prime}$, so $\omega_{0} \notin \partial \Omega^{\prime}$. It follows that $\sigma_{e}(T)=\partial \Omega^{\prime}$.

The inclusion $\Omega^{\prime} \subseteq \Omega_{0}$ follows from Subin's Theorem ([3], Cowen and Douglas). Also, it is easy to see that $\Omega_{0} \subseteq \Omega^{\prime}$. Hence $\Omega_{0}=\Omega^{\prime}$ and $\sigma_{e}(T)=\partial \Omega_{0}$. Q.E.D.

We now have proved the following.

(1.2) COROllary. Let $T \in \mathscr{B}_{n}^{\prime}(\Omega)$ and let $\Omega_{0}$ be as in Theorem 1.1. Then $\sigma_{e}(T)=$ $\partial \Omega_{0}$.

For the rest of our discussion let $G$ be a bounded, open, connected, nonempty subset of the complex plane $C$ and let $L^{2}(G)$ denote the $L^{2}$-space of (Lebesgue) area measure restricted to $G$. Denote by $L_{a}^{2}(G)$ the subspace of analytic functions 
belonging to $L^{2}(G)$. The Bergman space $L_{a}^{2}(G)$ is actually a closed subspace of $L^{2}(G)$ and thus is a Hilbert space. Let $T_{z}$ be the Bergman operator on $L_{a}^{2}(G)$ defined by $T_{z} f=z f$.

(1.3) Definition. A point $\lambda \in \partial G$ is said to be removable with respect to $L_{a}^{2}(G)$ if there exists an open neighborhood $V$ of $\lambda$ such that every function in $L_{a}^{2}(G)$ can be extended to an analytic function on $G \cup V$. The set of all points on $\partial G$ which are removable with respect to $L_{a}^{2}(G)$ is denoted by $\partial_{2-r} G$. The Bergman essential boundary of $G$, denoted by $\partial_{2-e} G$, is the set of all points of $\partial G$ which are not removable with respect to $L_{a}^{2}(G)$; so $\partial_{2-e} G=\partial G \sim \partial_{2-r} G$.

It is shown in [1] that $G \cup \partial_{2-r} G$ is an open subset of $\mathbf{C}$ and that $\partial_{2-r} G$ has zero area. Therefore it makes sense to consider $L_{a}^{2}\left(G \cup \partial_{2-r} G\right)$ and it is easy to see that $L_{a}^{2}(G)=L_{a}^{2}\left(G \cup \partial_{2-r} G\right)$, where the equality means that there is an obvious isometry between the two spaces. For $\lambda \in G \cup \partial_{2-r} G$, the linear functional on

$$
L_{a}^{2}\left(G \cup \partial_{2-r} G\right)=L_{a}^{2}(G)
$$

which takes $h$ to $h(\lambda)$ is bounded (see [2, p. 5]).

(1.4) Theorem. Let $G_{0}=\left\{\lambda_{0} \in \bar{G}: \operatorname{ran}\left(T_{z}-\lambda_{0}\right)\right.$ is closed and there exists a conjugate holomorphic $L_{a}^{2}(G)$-valued function $d(\lambda)$ defined on some neighborhood $V$ of $\lambda_{0}$ such that $\operatorname{ker}\left(T_{z}^{*}-\bar{\lambda}\right)=[d(\lambda)]$ for $\left.\lambda \in V\right\}$. Then $G_{0}=G \cup \partial_{2-r} G$. Equivalently $\sigma_{e}\left(T_{z}\right)=\partial_{2-e} G$.

Proof. First suppose that $\lambda \in G \cup \partial_{2-r} G$. Then it is easy to verify that the range of $T_{z}-\lambda$ is equal to the kernel of the linear functional on $L_{a}^{2}(G)=L_{a}^{2}\left(G \cup \partial_{2-r} G\right)$ which sends $h$ to $h(\lambda)$. In particular, the range of $T_{z}-\lambda$ is a closed subspace of $L_{a}^{2}(G)$ of codimension 1. Since $\operatorname{ker}\left(T_{z}-\lambda\right)=(0)$, we conclude that $T_{z}-\lambda$ is Fredholm (with index -1 ) and so $\lambda \notin \sigma_{e}\left(T_{z}\right)$. Thus $G \cup \partial_{2-r} G \subseteq \bar{G} \sim \sigma_{e}\left(T_{z}\right)$.

Next choose $\lambda_{0} \in G_{0}$. Let $h(\lambda)=(1, d(\lambda))$. If $h(\lambda) \equiv 0$ for $\lambda$ in a neighborhood of $\lambda_{0}$, then since $\lambda_{0} \in \bar{G}$, there exists $\lambda_{1}$ in $G$ such that $h\left(\lambda_{1}\right)=0$. This shows that $1 \in \operatorname{ran}\left(T_{z}-\lambda_{1}\right)$, or equivalently $\left(z-\lambda_{1}\right)^{-1}$ is in $L_{a}^{2}(G)$ and this is absurd. Therefore if $\lambda_{0} \in G_{0}$, then $h$ is holomorphic in a neighborhood of $\lambda_{0}$ and does not vanish identically there. Hence there exists $r>0$ such that $B\left(\lambda_{0}, r\right) \subseteq G_{0}$ and for $\lambda$ in $B\left(\lambda_{0}, r\right), h$ is holomorphic and $h(\lambda) \neq 0$ for $\lambda$ in $B_{0}=B\left(\lambda_{0}, r\right) \sim\left\{\lambda_{0}\right\}$.

Now for $\lambda$ in $B_{0}$ define $k(\lambda)=\overline{h(\lambda)}^{-1} d(\lambda)$. Obviously $k(\lambda), \lambda \in B_{0}$ has the same properties as $d(\lambda)$. Furthermore it is normalized such that $(1, k(\lambda))=1, \lambda \in B_{0}$. Let $\{e(\lambda)\}$ be the Bergman kernel (reproducing kernel) for $L_{a}^{2}(G)$. Then for $\lambda \in G \cap B_{0}, \operatorname{ker}\left(T_{z}^{*}-\bar{\lambda}\right)=[e(\lambda)]=[k(\lambda)]$. Hence $e(\lambda)=\overline{c(\lambda)} k(\lambda)$, where $c(\lambda)$ $\in \mathbf{C}$. By normalization, $1=(1, e(\lambda))=c(\lambda)(1, k(\lambda))=c(\lambda)$ and therefore $e(\lambda)=$ $k(\lambda)$. If $f \in L_{a}^{2}(G)$, then $f(\lambda)=(f, e(\lambda)), \lambda \in G$. Now define $\hat{f}(\lambda)=(f, k(\lambda))$, $\lambda \in B_{0}$. Clearly $\hat{f}(\lambda)=f(\lambda), \lambda \in B_{0} \cap G$. Hence $B_{0} \subseteq G \cup \partial_{2-r} G$. If $f \in L_{a}^{2}(G)=$ $L_{a}^{2}\left(G \cup \partial_{2-r} G\right)$, then $f \mid B_{0}$ is in $L_{a}^{2}\left(B_{0}\right)$ because $\partial_{2-r} G$ has zero area [1]. However, a function in $L_{a}^{2}\left(B_{0}\right)$ extends to $B$ [1]. Thus each function in $L_{a}^{2}(G)$ extends to be analytic on $B$. This shows that $\lambda_{0} \notin \partial_{2-e} G$. Hence $G_{0} \subseteq G \cup \partial_{2-r} G$.

We will show that $G_{0}=\bar{G} \sim \sigma_{e}\left(T_{z}\right)$. To see this let $\Omega=G^{*}$ and $T=T_{z}^{*}$. Then $T$ satisfies the hypothesis of Theorem 1.1 with $n=1$. Therefore $\sigma_{e}(T)=\partial \Omega_{0}$. It is easy 
to see that $\Omega_{0}=G_{0}^{*}$. Hence $\sigma_{e}\left(T_{z}\right)=\sigma_{e}\left(T^{*}\right)=\sigma_{e}(T)^{*}=\left(\partial \Omega_{0}\right)^{*}=\partial \Omega_{0}^{*}=\partial G_{0}$. Since $G_{0}$ is open, $\bar{G} \sim \sigma_{e}\left(T_{z}\right)=\bar{G}_{0} \sim \partial G_{0}=G_{0}$.

We have already shown that $G_{0} \subseteq G \cup \partial_{2-r} G \subseteq \bar{G} \sim \sigma_{e}\left(T_{z}\right)$; therefore $G_{0}=G \cup$ $\partial_{2-r} G=\bar{G} \sim \sigma_{e}(T)$. We conclude that $\sigma_{e}\left(T_{z}\right)=\partial_{2-e} G$. Q.E.D.

2. Weighted shifts in $A_{1}(G)$. For a connected open subset $G$ of the plane and $n$ a positive integer, let $A_{n}(G)$ denote the operators $S$ in $E(\mathcal{H})$ which satisfy

(a) $G \subseteq \sigma_{p}\left(S^{*}\right)^{*}$,

(b) $G \cap \sigma_{a p}(S)=\square$,

(c) $\sigma_{p}(S)=\square$,

(d) $\cap_{\lambda \in G} \operatorname{ran}(S-\lambda)=(0)$,

(e) $\operatorname{dim} \operatorname{ker}\left(S^{*}-\bar{\lambda}\right)=n, \lambda \in G$, and

(f) $\sigma(S)=\bar{G}$.

The space $A_{n}(G)$ is closely connected with the space $\varphi_{n}(\Omega)$. In particular it is easy to see that $A_{n}(G)=\left\{S: T=S^{*} \in \cup B_{n}^{\prime}\left(G^{*}\right)\right\}$.

A unilateral (bilateral) weighted shift on a Hilbert space $t \mathcal{H}$ is a linear map $T$ from $\mathcal{H}$ to itself given by $T e_{1}=w_{1} e_{1+1}$, where $\left(e_{1}\right)=\left(e_{1}\right)_{i=0}^{\infty}\left(\left(e_{i}\right)_{i=-\infty}^{\infty}\right)$ is an orthonormal basis for $\mathcal{H}$ and $\sup _{k}\left|w_{k}\right|<\infty$.

In his survey article, A. L. Shields [5] has investigated the properties of weighted shifts which we need in order to characterize the weighted shifts in $A_{1}(G)$. For a more detailed treatment of the subject and pertinent terminology see [5]. We now make a few observations.

Let $S$ be in $A_{n}(G)$ and note that $\sigma(S) \sim \sigma_{a p}(S)=\left\{\partial \sigma(S) \sim \sigma_{a p}(S)\right\} \cup\left\{[\sigma(S)]^{\circ}\right.$ $\left.\sim \sigma_{a p}(S)\right\}$. Because $\partial \sigma(S) \subseteq \sigma_{a p}(S)$ and $\sigma_{a p}(S)$ is closed (see Halmos [4, problem 62]), we conclude that $\sigma(S) \sim \sigma_{a p}(S)$ is an open set. Put $G_{S}=\sigma(S) \sim \sigma_{a p}(S)$; then $G \subseteq G_{S} \subseteq \sigma(S)$, so $\sigma(S)=\bar{G}=\bar{G}_{S}$. If $\lambda \in G_{S}$, then $\lambda \notin \sigma_{a p}(S)$. so $S-\lambda$ is bounded below. Therefore $S-\lambda$ has closed range. Also $\operatorname{ker}(S-\lambda)=(0)$, from which we conclude that $S-\lambda$ is semi-Fredholm. Now choose a sequence $\left\{\lambda_{k}\right\}$ in $G$ such that $\lambda_{k} \rightarrow \lambda$; then $\operatorname{ind}\left(S-\lambda_{k}\right) \rightarrow \operatorname{ind}(S-\lambda)$. Now for $S$ in $A_{n}(G)$ and $z \in G, S-z$ is a Fredholm operator and $\operatorname{ind}(S-z)=-n$. Therefore $\operatorname{ind}(S-\lambda)=-n$ and we conclude that $S \in A_{n}\left(G_{S}\right)$. Clearly $G_{S}$ is the largest open subset $G$ of the plane such that $S \in A_{n}(G)$.

If $A$ is any operator let $m(A)=\inf \{\|A f\|:\|f\|=1\}$. Then the following is true (see Shields [5]):

$$
\sup _{n}\left[m\left(A^{n}\right)\right]^{1 / n}=\lim _{n \rightarrow \infty}\left[m\left(A^{n}\right)\right]^{1 / n} .
$$

Let $r_{1}(A)=\lim _{n \rightarrow \infty}\left[m\left(A^{n}\right)\right]^{1 / n}$. If $A$ is invertible, then $r_{1}(A)=\left[r\left(A^{-1}\right)\right]^{-1}$. Also let $r(A)$ denote the spectral radius of $A$.

If $T$ is an injective unilateral weighted shift with weight sequence $\left\{w_{n}\right\}$, then we define $r_{2}(T)$ by $r_{2}(T)=\liminf _{n \rightarrow \infty}\left[w_{0} w_{1} \cdots w_{n-1}\right]^{1 / n}$. Thus $r_{1}(T) \leqslant r_{2}(T) \leqslant r(T)$.

In order to characterize the unilateral weighted shifts in $A_{1}(G)$, we first let $G=\left\{z:|z|<r_{1}(T)\right\} ;$ then

(a) $G \subseteq \sigma_{p}\left(T^{*}\right)[5$, Theorem 8(ii)],

(b) $G \cap \sigma_{a p}(T)=\square[5$, Theorem 6], 
(c) $\sigma_{p}(T)=\square[5$, Theorem 8(i)],

(d) If $f \in \cap_{\lambda \in G} \operatorname{ran}(T-\lambda)$, then $f \equiv 0[5$, Theorem (ii)], and

(e) $\operatorname{dim} \operatorname{ker}\left(T^{*}-\bar{\lambda}\right)=1, \lambda \in G[5$, Theorem 8]. However, $\sigma(T)$ might not be equal to $\bar{G}$ unless $r_{1}(T)=r(T)$, in which case $r_{1}(T)=r_{2}(T)=r(T)$.

Next let $T$ be any unilateral weighted shift in $A_{1}(G)$. Then $\bar{G}=\sigma(T)=\{z$ : $|z| \leqslant r(T)\}$, so $G \subseteq\{z:|z|<r(T)\}$. Since $G \cap \sigma_{a p}(T)=\square$, we have $G \subseteq\{z$ : $\left.|z|<r_{1}(T)\right\}$. It follows that $\bar{G} \subseteq\left\{z:|z| \leqslant r_{1}(T)\right\}$. But $\bar{G}=\{z:|z| \leqslant r(T)\}$, and from this one obtains that $r_{1}(T)=r(T)$. Now $G_{T}=\operatorname{int}\left[\sigma(T) \sim \sigma_{a p}(T)\right]=\{z:|z|<$ $r(T)\}$.

Note. If $T$ is a unilateral weighted shift in $A_{1}(G)$, then the set $G_{T}$ is actually independent of $T$. To see this let $T$ and $T^{\prime}$ be unilateral weighted shifts in $A_{1}(G)$. Then $\sigma(T)=\sigma\left(T^{\prime}\right)=\bar{G}$, so $r(T)=r\left(T^{\prime}\right)$. We have also shown that $r(T)=r_{1}(T)$ and $r\left(T^{\prime}\right)=r_{1}\left(T^{\prime}\right)$. Therefore $r(T)=r\left(T^{\prime}\right)=r_{1}\left(T^{\prime}\right)=r_{1}(T)$. Hence $G_{T}=G_{T^{\prime}}$.

We have therefore proved the following.

(2.1) THEOREM. Let $T$ be a unilateral weighted shift. Then $T$ is in $A_{1}(G)$ if and only if $\bar{G}=\{z:|z| \leqslant r(T)\}$ and $r(T)=r_{1}(T)$.

If $T$ is an injective bilateral weighted shift with weight sequence $\left\{w_{n}\right\}$ then define $r_{2}^{+}(T)=\liminf _{n \rightarrow \infty}\left[w_{0} \cdots w_{n-1}\right]^{1 / n}, r_{3}^{+}(T)=\limsup _{n \rightarrow \infty}\left[w_{0} \cdots w_{n-1}\right]^{1 / n}, r_{2}^{-}(T)$ $=\liminf _{n \rightarrow \infty}\left[w_{-1} \cdots w_{-n}\right]^{1 / n}, r_{3}^{-}(T)=\lim \sup _{n \rightarrow \infty}\left[w_{-1} \cdots w_{-n}\right]^{1 / n}$. Then $r_{1}^{-} \leqslant r_{2}^{-}$ $\leqslant r_{3}^{-} \leqslant r^{-}$and $r_{1}^{+} \leqslant r_{2}^{+} \leqslant r_{3}^{+} \leqslant r^{+}$.

In order to characterize the bilateral weighted shifts in $A_{1}(G)$, we first let $G=\{z$ : $\left.r_{1}(T)<|z|<r(T)\right\}$ and assume $r_{1}=r_{1}^{-}=r^{-}, r_{1}^{+}=r^{+}=r\left(r_{1}<r\right)$. It follows that

(a) $G \subseteq \sigma_{p}\left(T^{*}\right)$ [5, Theorem 9(iii)],

(b) $G \cap \sigma_{a p}(T)=\square[5$, Theorem 7],

(c) $\sigma_{p}(T)=\square[5$, Theorem 9(ii)],

(d) if $f \in \cap_{\lambda \in G} \operatorname{ran}(T-\lambda)$, then $f \equiv 0$ [5, Theorem 10'(ii)],

(e) $\operatorname{dim} \operatorname{ker}\left(T^{*}-\bar{\lambda}\right)=1, \lambda \in G[5$, Theorem 9(i)],

(f) $\sigma(T) \subseteq \bar{G}[5$, Theorem $5($ a) $]$.

Next let $T$ be any bilateral weighted shift in $A_{1}(G)$. If $T$ is not invertible, then $\sigma(T)=\{z:|z| \leqslant r(T)\}$. Since $G \cap \sigma_{a p}(T)=\square, r^{-}<r_{1}^{+}$and $\sigma_{a p}(T)=\left\{r_{1}^{-} \leqslant|z| \leqslant\right.$ $\left.r^{-}\right\} \cup\left\{r_{1}^{+} \leqslant|z| \leqslant r^{+}\right\}$. Now $r_{1}^{-} \leqslant r^{-}<r_{1}^{+} \leqslant r^{+}$, so $G \subset\left\{|z|<r_{1}^{-}\right\} \cup\left\{r^{-}<\right.$ $\left.|z|<r_{1}^{+}\right\}$. From this we get $\bar{G} \subseteq\left\{|z| \leqslant r_{1}^{-}\right\} \cup\left\{r^{-} \leqslant|z| \leqslant r_{1}^{+}\right\}$. But $\bar{G} \subseteq\{|z| \leqslant$ $r(T)\}$, so $r_{1}^{-}=r^{-}=r_{1}^{+}=r^{+}$. Equivalently $r_{1}=r$. Thus $r_{1}^{-}=r_{2}^{-}=r_{3}^{-}=r=r_{1}^{+}=$ $r_{2}^{+}=r_{3}^{+}$. It follows that $\sigma_{p}\left(T^{*}\right)=\{|z|=r\}$. Therefore (a) does not hold. We have shown that if $T$ is a bilateral weighted shift in $A_{1}(G)$.it is necessary that $T$ be invertible. In this case $\sigma(T)=\left\{r_{1} \leqslant|z| \leqslant r\right\}, \sigma_{a p}(T)=\left\{r_{1}^{-} \leqslant|z| \leqslant r^{-}\right\} \cup\left\{r_{1}^{+} \leqslant\right.$ $\left.|z| \leqslant r^{+}\right\}$, and $r_{1}^{-} \leqslant r^{-} \leqslant r_{1}^{+} \leqslant r^{+}$. Since $G \cap \sigma_{a p}(T)=\square$, it is easy to see that the only possibility is $r_{1}^{-}=r^{-}$and $r_{1}^{+}=r^{+}$. So $\sigma_{a p}(T)=\{|z|=r\} \cup\left\{|z|=r_{1}\right\}$. We also have $G_{T}=\operatorname{int}\left[\sigma(T) \sim \sigma_{a p}(T)\right]=\left\{z: r_{1}<|z|<r\right\}$.

Note. It is easy to see that $G_{T}$ does not depend on $T$. We have thus proved the following.

(2.2) THEOREM. If $T$ is a bilateral weighted shift, then $T$ is in $A_{1}(G)$ if and only if $\bar{G}=\left\{z: r_{1}(T) \leqslant|z| \leqslant r(T)\right\}, r_{1}^{-}=r^{-}$and $r_{1}^{+}=r^{+}$. 


\section{REFERENCES}

1. S. Axler, J. Conway and G. McDonald, Toeplitz operators on Bergman spaces, Canad. J. Math. 34 (1982), 466-483.

2. S. Bergman, The kernel function and conformal mapping, 2nd rev. ed., Math. Surveys, no. 5, Amer. Math. Soc., Providence, R. I., 1970.

3. M. Cowen and R. Douglas, Complex geometry and operator theory, Acta Math. 141 (1978), 187-261.

4. P. Halmos, A Hilbert space problem book, Van Nostrand, Princeton, N. J., 1967.

5. A. Shields, Weighted shift operators and analytic function theory, Topics in Operator Theory, Math. Surveys, no. 13, Amer. Math. Soc., Providence, R. I., 1974, pp. 49-128.

DEPARTMENT OF MATHEMATICS, INDIANA UNIVERSITY, BLOOMINGTON, INDIANA 47405

Current address: Department of Mathematics and Statistics, University of Calgary, Calgary, Alberta, Canada T2N IN4 